needs all the courage possible. There is almost no limit to which he may not go to obtain the results that he starts out to secure in these cases. We may begin with the idea of a simple mastoid operation and conclude, after a few steps, that we will have to perform a radical mastoid operation, and after we have done that we may not yet be satisfied. I will never forget the feelings I experienced in the clinic of Professor Schwartze, when he began what he himself considered a simple mastoid operation; following this he did the radical one; then going down in the neck he made a ligation of the jugular rein; then going up above again he found an epidural abscess, and then going into the lateral sinus and cutting the external vein, he syringed from the mastoid back into the neck. That was carried on two or three weeks with final success. It was an operation that anybody would be proud of doing, and it shows what we may do if we have the courage of our convictions.

DR. D. M. Greene, Grand Rapida, Mich.-Dr. Whiting's paper is on a subject to which I have given a great deal of atten tion, and in which I have been deeply interested. Fortunately I have had but one case of sinus thrombosis, and unfortunately no operation was done on the mastoid or otherwise, but the autopsy revealed a sinus thrombosis. I made a diagnosis of mastoid disease and wanted to do an operation, but at that time, about ten years ago, it was rather a new thing to operate iwithout external swelling, and I was opposed by the physicians in cousultation. The patient died as the result, from metastatic abscesses.

$I$ desire to emphasize the following point. Tenderness and swelling along the line of the jugular vein, was not to be felt, and there was no choied disc and no swelling above the eye, none of the signs and symptoms which the Doctor has enumerated as a diagnosis of that condition, except that after the metastatic abscesses had formed in the body, a chain of swelling occurred along the juyular vein.

Dr. A. J. Timrefaran, Columbus. Ohia-1 wouId like Dr. Whiting to tell us whether he has ever, in noting the symptomatology of these cases, known of patients who complained of pain in the shoulder-blade.

Dr. Fred Whiting, Now York City-In reply to the last speaker I must say that I have noticed that symptom once, but only in a fatal case, and the patient also complained of pain in the anterior pectoral region. It was a case which subsequertly proved to be a septic pneumonia and a pleuropneumonia in addition at that point. In regard to $\mathrm{D}^{\prime}$. Greene's statements that the cord-like cordition of the internal jugular vein on the affected side did not appear until the netastatic abscesses had appeared, that is quite common. Probably that patient did not have a thrombis so complete jas to entirely close the

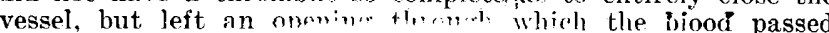
downward, and the septic material then was carried along the vein down into the lungs, so you had metastatic abscesses and then the phlebitis recame extensive and you then had septic phlebitis extending so you could make out tirese enlargements. As to the inquiry as to whether the rein itself sometimes be comes so involved in phlebitis as to he unrecognimable in the neck. that is a condition $I$ have not met. Schwartze mention. a case in which he cculd find no remnant of the rein and had only an abscess cavity in the neck. Knapp also reports a case in which he made an autopsy and endeavored to find the jug. ular in order to make the operation such as he would and tie of the jugular, and he could find nothing except what he characterized as a minute thread. In that case there had been very extensive cicatrical obliteration. I have never found obliterat ion of the vein so that it was not easily found. As a rule the vein collapses below the elot. and while it is sometimes somewhat difficult to find, if the man is well acquainted with the anatomy of the part, he can find it quite readily.

\section{DIABFTES MELLITUS.*}

BY J. M. ALI.EN, A.M., M.D., ILT.D. L.IBERTY, MO.

Diabetes mellitus is a disease in the beginning of perverted nutrition, caused either by hereditary, functional or organic disease. There is some testimony that it may be communicated from one person to another. It also follows syphilitic lesions near the fourth ventrick of the brain. Tater pathologic changes may occur in one or more of the following organs: pancreas, liver, heart, and kidneys, also in the nerve centers of the

* Presented to the Section on Materia Medica. Pharmacy and Therapentics, at the Fiftieth Annual Meeting of une American Medical Association, held at. Columbus. Ohio, June 6-9, 1899. cardiac and solar plexus. 'The perversion consists of the entrance in to the blood of an abnormal quantity of saccharine matter. This saccharine matter practically acts as a strong diuretic by producing hyperemia of the kidneys, which results in increased functional action manifested by the passage of large quantities of urine loaded with saccharine matter depuriated from the blood. While this perverted functional action of the kidneys is largely in excess of normal, it must be considered as a conservative force removing from the blood matter which would be injurious to normal tissue metamorphosis.

If the proposition is true that the etiologic factor in this disease is a perversion of nutrition, we should begin its study from two sources: the food matter and the shylopoietic viscera.

It has been abundantly proved that the eating of large quantities of sugar does not necessarily produce this disease, but it may increase the sugar in the urine after the discase is established.

The ultimate of the carbohydrates, and a small portion of the nitrogenous substances, results in the production of saccharine matters in the process of digestion, which, with normal metabolic changes referred to, occur below the pylorus, hence it is unnecessary for us to give attention to the digestive tract above this valve.

The various experiments and pathologic investigations of the part the pancreas might play in the production of this disease is far from being conclusive. The weight of evidence, pro and con, is against the pancreas being an etiologic factor in the production of this disease. The same is true in regard to the liver and the heart, therefore, it would be a work of supererogation for me to bring forward the authorities and reasons on this point, because our text-books and literature abound with them.

It is not so clear to my mind as to the rôle the central and sympathetic nervous systems play in its etiologr, because Benard and others have established beyond question that irritation or disease of the fourth ventricle is uniformly followed by sugar in the urine, but this does not follow in the experiments or pathologic changes in any of the other above-named organs. Nor are any of the known diseases of the liver, either functional or organic, ever followed or associated with diabetes. The same is true of the heart. In two postmortems held by me, the pancreas was practically destroyed, and no diabetes was present.

A number of years ago my attention was attracted to the character of food prescribed for diabetic patients by the fact that it excluded all the food digested and assimilated in the duodlenum and the other small intestines. And at that time neither physiology nor pathology gave any reason for this course. True, we know that the carbohydrates and saccharine matters of the food were digested and assimilated in this tract of the intestines. The withholding of these, I could easily recognize, gave these organs rest by lessening physiologic functional hyperemia. But the questiton suggested to my mind was: Why do this, unless there are organic diseases of the duodenum and jejunum? I believed then, as I do now, that the beneficial effects of withdrawing carbohydrates and saccharine matter from diabetic patients was beneficial only because of disease of the duodenum. With these views before me I began a careful examination of every case of diabetes that presented itself, either in my clinic or private practice, for chronic inflammation and ulceration of the duodenum. Since 
that time I have had twenty-six cases of diabetes that I am sure were preceded by chronic inflammation and ulceration of the duodenum. All of these were carefully and thoroughly examined for disease of the heart, liver and pancreas, but none was found. Seven of these cases 1 made careful post-mortems of, and found chronic inflammation and ulceration of the duodenum, but no Iesion of either pancreas, heart or liver, except in one, where the liver was a little pale in color. In three of them there were lesions near the origin of the pneumogastric nerve which I ascribed to reflex irritation from the duodenum.

In 1818 there was a patient admitted to the Kansas City Hospital, with paralysis of the upper and lower extremities which had existed for five days. He gave the history of having had diabetes for about six months, also that he had had syphilis eighteen months before entrance, which had followed its normal course. $\mathrm{He}$ died twelve hours after admittance. Post-mortem examination revealed a clot in the spinal canal near the center of the last cervical and the first dorsal, the result of degeneration of the arterial coats, and gummata in the fourth ventricle of the brain; the other organs of the body were in a healthy condition, including the duodenum.

As to the value of opium, after studying its therapeutic effects, which are, for a time, to arrest secretion, after which, under its use, secretion returns to a normal condition, as is demonstrated in the use of it in large quantities in peritoneal inflammations and opium habituates, I have failed to see why it could possibly be beneficial in diabetes mellitus, except its controlling effect on reflex irritation, which does not require the immonse doses that are given. This fact was strongly illustrated in two cases of diabetes mellitus following pachymeningitis, which fell under my care. I used opium in these cases in increasing large doses, yet it had no effect whatever, neither reducing the quantity of the sugar in the urine nor the quantity of the urine secreted.

When it is remembered how abundantly the duodenum is furnished with blood, and how intimately it is connected with the central nervous system by the par ragum, and to the sympathetic through the solar plexus; by the splanchnic with the thoracic centers and filaments from the spinal cord, also with the plexus of Meissner in the mucous membrane, and that of Auerbach between the muscular coats; and how abundantly it is studded with the glands of Lieberkuhn and Brunner, the secretions of which have much to do with the metabolic changes of our food; and coupling this with it being the receptacle for the secretion of the bile and pancreatic fluid, we are forced to recognize that it is an organ of great importance in the digestive and assimilative process, possibly as important as the stomach itself.

$\mathrm{By}$ its anatomic location and relation to the stomach, it becomes the receptacle for all the matter that passes through the pylorus, and in those who are suffering from either functional or organic disease of the stomach much of this matter is highly irritating. Its extensive and various connections make it the great reflex center of the alimentary canal. This is demonstrated by the fact that nearly all severe burns produce inflammation and ulceration of this organ. This organ is also exposed to the inflammatory diseases of the intestinal canal, particularly dysentery, typhoid fever, and oceasionally the exanthematous diseases.

I hope I shall be excused for making this digression, becanse of the scarcity of medical literature on the sub- ject, and it enables me to bring before you the importance of the functions of this organ, and the frequency with which it is exposed to disease.

It is fair to assume that chronic inflammation and ulceration of this organ would materially affect the secretion of its glands, and possibly its accessories, thereby arresting those metabolic changes in our food whence the saccharine matter is derived, and rendering them unfit to be converted into glycogen, so that by natural selection they are taken up by the absorbents and veins and carried to the kidneys to be depurated by them from the blood.

As all the cases of diabetes that have fallen under my care have been more or less of a neuropathic diathesis, with high reflex sensibilities, and recognizing the duodenum as the great center of reflexes in the alimentary canal, the question has often presented itself to my mind as between the explanation given above in regard to the metabolic changes in the food as an etiologic factor and peripheral irritation, producing reflex irritation, affecting the nutrition of the nerve-center that presides over the conversion of starchy matter into saccharine matter, and as to which is the etiologic factor in diabetes.

I am free to admit that the tendency of my mind, taking into consideration the origin and distribution of the par vagum and the connection of the duodenum with the sympathetic nervous centers, is to conclude that peripheral irritation is the more probable factor, because Brown-Séquard and others have well established that reflex irritation is sufficient to arrest and prevent secretion; and, if continued long enough, to produce structural change of tissue.

Basing my treatment on the philosophy of etiology and pathology as given, it is as follows:

1. Allow the patient to eat only such foods as can be digested by the stomach, giving the duodenum rest, and thereby preventing physiologic hyperemia. This continued for a time will reduce the hyperemia to a point so small and feeble as not to be a source of irritation to the inflamed structures.

2. Tse intestinal germicides, the value of which, in my expericnce, is in the order given: creosote, sulphocarbolates of zine and soda, hyposulphite of soda, eucalyptol, menthol, oil of cinnamon and sulphid of calcium, salol, betanapthol, carbonate of guaiacol. I have found the pulvis hydrastis canadensis, combined with salol, an efficient stimulant and alterative to the mucous membrane. To control reflex irritation, I use 1-15-1-10 gr. of morphin three times daily, or aqua chloroformi, a dessertspoonful three times daily.

All of these cases suffer more or less with anemia of the nerve centers: To prevent this I give 1-60-1-40 gr. strychnin nitrate three times a day, or an equivalent amount of nux vomica. As a tonic I use either the bitter tonics or the phosphates of lime and soda, or the wine of the phosphoglycerate of lime (Chapteau). The ferruginous preparations and the cod-liver oils should be excludied.

Many cases of diabetes have hyperacidity and hypersecretion caused by reflex irritation originating in the duodenum, so great that when the chyme passes through the pylorus it can not be rendered sufficiently alkaline by the secretions in the duodenum to undergo normal metabolism.

This condition is always associated with an increased amount of sugar in the urine. To render the chyme less acid we give from 20-30 grs. of potassium bicarbonate, largely diluted in water, just after eating. Its ben- 
eficial effects are at once manifested by a decreased amount of sugar in the urine and in the amount of urine secreted; it also makes it far less irritating to the duodenum, thereby decreasing the reflex trouble. The salicylate of soda is recommended for the same purpose, but in my hands the results have not been so desirable; the only advantage it could have over potasoium bicarbonate would be its slight germicidal properties; but this effect is not needed, for the reason that I am giving better germicides.

Another combination of remedies which I have recently used with apparent benefit is as follows:

R. Creosote ......................... gtts. $1 / 3$

Tinct. nucis vomicæ................gtts. $\mathrm{x}$

M.

Saw palmetto....................

In the saw palmetto we have something of a tonic as well as a stimulant to the mucous membrane, thereby lessening retrograde metamorphosis of this tissue.

As a matter of course the environments of the patient should be hygienic, with plenty of fresh air, also baths and massage of the skin every other day, and no mental nor physical work.

$A$ large majority of the cases of diabetes which $I$ have treated have been in my clinic of the University Medical College, Kansas City, Mo. Only three of these remained with me ten or twelve months, when they were discharged cured. Two cases recovered in my private practice. The remaining number I was unable to follow, so I can not give results. I think I do not say too much when I say all of them were benefited, as indicated by a decrease in the amount of secretion of urine and the amount of sugar present.

Excluding heredity as a factor, and beginning early before pathologic changes have occurred, I should feel hopeful of favorable results.

\section{CIINICAL NOTIS ON DIGITOXIN.* BY JOHN P. SAWYER, M.D. CLEVELAND, OHIO.}

Among the few really indispensable drugs which we have, digitalis would be placed in the very front rank by most medical men, and this is true in spite of the fact that its use is attendea by certain disadvantages, and that these disadvantages are not regularly to be estimated, owing to the complex nature of the constituents of the drug, and the varying proportions in which these are present in the crude leaves. The various alkaloids and glucosids, obtained from the crude drug, have not been widely known because of technical difficulties in preparation, and have not made their way into general favor. Moreover, the exceeding smallness of the dose, and the considerable risk of cumulative effect have added to the distrust with which most practitioners have looked on these drugs. But the evident gain from the use of a definite active principle is so great that determined efforts have been continued through years. and there is now a considerable mass of cumulative evidence very favorable to the use notably of digitoxin. And I bring it to your notice here because I have found in an increasingly free use of the drug that it is more speedy. more certain than digitalis; that it is efficient in most cases where digitalis is at all likely to succeed-and this may be well inferred from the fact that digitoxin confers on the leaves a large part of their ordinary effectiveness-and, moreover, in a number of cases I have found

* Presented to the Section on State Medicine, at the Fiftleth Annual Meeting of the American Medical Association, held at Columbus, Ohio, June 6-9, 1899. digitoxin efficient to a remarkable degree, when digitalis had failed utterly in an ordinary method of administration, and when also a long list of other allied heart tonics had been employed successfully. In fact, I am now using digitoxin in a majority of cases under my observation which present any indication for the use of digitalis.

I will especially refer to an article by $W_{\text {enzel }}{ }^{1}$, in which he reports the use of digitoxin under Unverricht's directions in the Madgeburg hospital. This is the more noticeable as within a few weeks Unverricht has siated that he has in these intervening years made a continually increasing use of digitoxin, until now it has nearly displaced digitalis.

The remedy has been administered in solution with glycerin, alcohol, chloroform diluted in water. It has been inade use of hypodermically, and has largely been given per rectum. Many writers refer to its irritating properties on the stomach, but this is true particularly of earlier articles, and as the dose has been diminished, there are apparently fewer reports of unpleasant results on the digestive canal. I have as yet to see in the small dose I employ the first symptom of irritation in the stomach, and I have not found it necessary to resort to any other means of medication than by capsules or tablets given by the mouth.

The desirable effect sought to be gained by the adminis!ration of digitalis is an increase of arterial pressure, cisually with diminution of pulse-rate; while on the other hand we seek to avoid continuance of high arterial pressure with quickening rate, heightened blood pressure with irregularity, and as the extreme the rapid sinking of blood pressure with sudden, cessation of heart action, and death. The great weight of experience is that digitoxin accomplishes this first desirable indicatjon within from twelve to twenty-four hours, and the result once accomplished is maintained for a surprisingly long period of time.

My own observations coincide entirely with these statements, and I have a sphygmogram taken from a case of a wonan 30 years of age, with a rapid pulse of low censior, slightly irregular, with heart apex in the mammary line in the fifth interspace, with a systolic murmur heard at the apex and toward the base, with accentuation of the pulmonary second sound, complaining of shortness of breath on slight exertion, complaining of a dry hacking cough after exertion, and particularly annoyed by it on jying down at night - so much so that she was unable to sleep for several hours. She had also noticed a considerable diminution in the amount of urine. I directed one-eighth of a milligram, about 1/500 grain, of digitoxin three times a day until the pulse was less than 80 , and directed her to report in forty-eight hours. She took two doses the first day, and had very little cough at night; one dose the following morning, and then finding her pulse less than 80, took no more until I had seen luer again. I told her to take one dose each evening and report again in forty-eight hours. when I took a second tracing. which shows a decidedly improved condition of arterial pressure, pulse wave far better sustained, and the rate reduced to normal. The patient took but three or four doses, and has been entirely relieved of the symptoms of insufficiency with which she came, and has noticed a marked increase in the quantity of urine.

This learls me to the statement that I have repeatedly noted the favorable diuretic effect in digitoxin. What $I$ hare seen in this one case. I have seen in more than a

\footnotetext{
I Centralbl. f. Inn. Med., 1885.
} 